\title{
Assessment of Abdominal Pain Through Global Outcomes and Recent FDA Recommendations in Children. Are We Ready For Change?
}

\author{
Saeed Mohammad, MD1, Carlo Di Lorenzo, MD², Nader N. Youssef, MD³, Adrian Miranda, \\ MD ${ }^{4}$, Samuel Nurko, MD ${ }^{5}$, Paul Hyman, $\mathbf{M D}^{6}$, and Miguel Saps, $\mathbf{M D}^{1}$ \\ ${ }^{1}$ Division of Pediatric Gastroenterology, Hepatology and Nutrition, Ann \& Robert H Lurie \\ Children's Hospital of Chicago, Northwestern University, Feinberg School of Medicine, Chicago, \\ IL
}

2Division of Gastroenterology, Nationwide Children's Hospital, Columbus, $\mathrm{OH}$

${ }^{3}$ Digestive Healthcare Center, Hillsborough NJ

${ }^{4}$ Division of Pediatric Gastroenterology, Hepatology and Nutrition, Medical College of Wisconsin, Milwaukee, WI

${ }^{5}$ Center for Motility and Functional Gastrointestinal Disorders, Children's Hospital Boston, Boston, MA

${ }^{6}$ Division of Pediatric Gastroenterology, Children's Hospital of New Orleans, New Orleans, LA

\section{Abstract}

Objectives-Irritable bowel syndrome is a multi-symptom construct with abdominal pain (AP) acting as the driving symptom of patient reported severity. The FDA considers a greater than $30 \%$ decrease in AP as satisfactory improvement, but this has not been validated in children. We investigated the correspondence of two measures for AP assessment, $230 \%$ improvement in AP and global assessment of improvement.

\begin{abstract}
Methods-Secondary analysis of data from 72 children who completed a randomized clinical trial for abdominal pain-associated functional gastrointestinal disorders. Children completed: 1daily assessment of AP intensity, 2-Functional Disability Inventory (FDI), 3-questions regarding pain's interference with activities and 4-two global assessment questions. We measured the extent to which $\geq 30 \%$ improvement of AP and global assessment questions correlated with each other and with disability.
\end{abstract}

Results-The global questions correlated with each other ( $r=0.74 ; \mathrm{p}<0.0001)$ and with a $230 \%$ improvement in AP $(\mathrm{p}<0.01)$. Global outcomes: Satisfaction with treatment was inversely related to the child's report of interference with activities $(\mathrm{p}<0.01)$ and symptom relief was positively associated with $\geq 30 \%$ improvement in FDI scores $(\mathrm{p}<0.009)$. Thirty percent change in FDI scores

Corresponding Author: Saeed Mohammad, MD, 225 E Chicago Ave, Box 65, The Ann \& Robert H. Lurie Children's Hospital of Chicago, Chicago, IL 60611, Tel: 312-227-4087, smohammad@luriechildrens.org.

Conflicts of Interest: The authors have no conflicts of interest to declare.

Financial Disclosure: Nothing to disclose 
was associated with global questions of symptom relief and $(\mathrm{p}=0.009)$ but not with satisfaction with treatment $(\mathrm{p}=0.07)$. The association of AP improvement with interference with activities $(\mathrm{p}=0.14)$ or change in FDI scores $(\mathrm{p}=0.27)$ did not reach significance.

Conclusions-Currently used global assessments are significantly associated with decreased pain intensity, decreased interference with daily activities, and a $\geq 30 \%$ change in FDI scores whereas recommended $30 \%$ improvement in pain intensity is not as comprehensive.

\section{Keywords}

Irritable Bowel syndrome; Pediatric Gastroenterology; Symptom score; Outcomes

\section{Introduction}

Abdominal pain (AP) associated-functional gastrointestinal disorders (FGIDs) including Irritable Bowel Syndrome (IBS), represent a group of multi-symptom constructs with AP acting as their driving symptom[1]. The diagnosis of AP associated-FGIDs is based on patient report of clinical symptoms. Physicians rely on patient's reports to establish disease severity, develop treatment plans and assess outcomes. Patient reported outcomes (PROs) are also essential to establish the efficacy of new drugs to be introduced to the market. Selection of PROs in the adult population has been hampered by the paucity of data to support their reliability and validity and there is no agreement on which PRO's are best. Selection of an outcome measure in pediatrics is even more complicated as developmental issues may limit the understanding of outcome measures used in adults. The Pediatric Initiative on Methods, Measurement and Pain Assessment in Clinical Trials (PedIMMPACT), consensus statement by academic researchers, representatives of government funding and regulatory agencies, and the pharmaceutical industry recommended the assessment of eight core domains in clinical trials of pediatric chronic pain including pain intensity, global judgment of satisfaction with treatment, sleep, physical and role function, and functional disability[2]. Although, this consensus does not exclusively address the study of children with gastrointestinal symptoms the study of these domains may also apply to children with FGIDs. Sleep problems and school absenteeism are common in children with functional AP[3]. Altered sleep has been shown to be associated with functional disability and factors other than severity of gastrointestinal symptoms determine school absenteeism in children with FGIDs. [4]. Landmark studies leading to the approval of medications for IBS have used "relief of symptoms" and "overall improvement" as PROs [5, 6]. Similar PROs have also been used in randomized clinical trials in children[7]. In 2010, the Food and Drug Administration (FDA) published a guidance document on the clinical evaluation of products for the treatment of IBS[8]. In these guidelines, the FDA criticizes some of the previously used PRO measures for the absence of sufficient qualitative research to support their validity "in a well-defined and reliable way" and made its own recommendations on optimal PRO's. These guidelines no longer support the use of global changes in a patient's IBS symptoms as PRO's. The FDA also questioned the reliability of overall assessment of change as it relies on recall of the patients' previous state, the inability to quantify intensity of current symptoms, and the presumed variability of interpretation among patients of the concept of "adequate" and "satisfactory" relief. Based on these 
limitations, the FDA recommended the development of multi-item PRO instruments that assess all the clinically relevant signs and symptoms of IBS. Additionally, they recommended the quantitative assessment of two interim co-primary endpoints for IBS one for pain ( $230 \%$ improvement) and the other for altered bowel habits[8]. The particularities of conducting clinical trials in children may limit the application of these guidelines. There are no widely accepted validated tools to assess stool form in children [9] and the child's ability to recall pain episodes is limited[10]. Although $230 \%$ improvement in pain intensity scores has been already used in one pediatric clinical trial [11], this endpoint has never been formally studied in children and there is no research to substantiate that changes in pain intensity should substitute the global outcomes measures.

Thus, our goal was to investigate the performance of the global PROs as recommended by the Rome II Committee and the FDA recommendations of improvement in pain intensity. We conducted a secondary analysis of an existing large database from the largest drug clinical trial in FGIDs in children [7]. The trial had a low attrition rate and was considered to be well designed and to have a low risk of bias[12]. The primary outcomes of this multicenter randomized clinical trial followed the recommendations of the Rome II Committee (patients' overall assessment of satisfactory symptom relief and satisfaction with treatment).

Changes in pain intensity were calculated based on children's daily report of symptoms. Daily diaries are considered the gold standard in pediatric clinical trials because frequent assessment of symptoms minimizes recall bias. These data thus allow us to compare results from global outcomes with a more specific PRO on AP.

We specifically aimed to measure the relation between the global questions and $\geq 30 \%$ improvement in AP with clinically meaningful outcomes.. We compared our results with a validated measure of disability in children the Functional Disability Index (FDI). A change of $\mathbf{3 0 \%}$ change in FDI scores was used as a positive outcome measure based on studies demonstrating $230 \%$ change in numerical rating scores as a clinically important difference in adults with chronic pain [13]. The selection of the FDI [14] as measure of physical functioning in children was based on the PedIMMPACT recommendations[2].

\section{Materials and Methods}

This study is a sub analysis of the database of a multicenter randomized placebo controlled trial designed to assess the efficacy of amitriptyline in children with AP associated-FGID. (Rome II criteria) This included 50.7\% with IBS, $42.4 \%$ with functional abdominal pain and $6.9 \%$ diagnosed with functional dyspepsia. For the purpose of this study we analyzed the available data of all children who returned all the daily diaries, responded to the global outcome measures at the end of the study and completed the FDI and disability questionnaires. The methods, study questionnaires and results of the study have been described in detail previously [7]. 


\section{Data collection}

Briefly, children completed diaries each night throughout the 4 weeks of the trial Daily diaries included information on AP and disability and a weekly average of the pain was calculated by dividing the sum of AP intensity by the number of days reported. Pain was assessed using a $100 \mathrm{~mm}$ Visual Analog/Likert scale with 5 anchors at $0 \mathrm{~mm}$ (no pain), $25 \mathrm{~mm}$ (little pain), $50 \mathrm{~mm}$ (medium pain), $75 \mathrm{~mm}$ (large pain) and $100 \mathrm{~mm}$ (worst possible pain). Patients missing more than 5 of 7 days were not considered for calculation and were excluded $(n=2)$ At the end of the trial, the children completed validated age appropriate psychological and disability questionnaires, questions on the extent to which pain interfered with their activities (Did pain interfere with daily activities such as going to school, playing or sleeping? Yes/No) and two global questions to assess the primary outcomes of the study. Global assessment questions followed the Rome II group recommendations on the design of clinical trials [15], and were based on clinical trials of two drugs that were originally approved by the FDA for the treatment of adults with IBS[5, 6]. Global assessment questions were: 'How well did the medication relieve your pain? Excellent, Good, Fair, Poor, Failed" (satisfaction with treatment) and "Overall how do you feel your problem is? Worse, Same or Better" (symptom relief). Disability was assessed through a validated, wellestablished commonly used pediatric questionnaire, the FDI. The FDI is a measure of the degree to which children experience difficulty in physical and psychosocial functioning due to their physical health status that includes ratings on ability to complete the school day, gym class, playing sports, and falling and maintaining sleep[14, 16-18]. Respondents are asked to rate on a 5-point Likert scale how much physical difficulty was perceived for a variety of everyday activities. The lower the score, the better is the patients' function. All children in the study had no other sources of physical disability other than those related to the AP-FGIDs.

\section{Data Analysis}

For the purpose of the current study, we have analyzed available data of self-report daily dairies and end of study questionnaires of children who completed the clinical trial. Improvement in pain intensity was calculated by establishing the difference in the mean of reported AP intensity $(\mathrm{mm})$ between the initial week and last week of daily diaries. The responses to both global assessment questions were analyzed as binary outcomes.

Satisfaction with treatment (excellent or good vs. fair, poor or fail) and satisfactory symptom relief (better vs. same or worse), We analyzed the correlation between the data from daily pain diaries, end of study disability questionnaires and changes in FDI scores with the responses to the global assessment questions, the FDA recommended clinical endpoint: $\geq$ $30 \%$ improvement in pain intensity and our own experimental endpoint: $\geq 50 \%$ improvement in pain intensity. The $\geq 50 \%$ change in pain intensity was chosen in an attempt to increase the sensitivity of this measure and the results are provided in Tables 1,3 and 4 . Strength of positive correlation was defined as: negligible 0.01-0.19; weak 0.20-0.29; moderate $0.30-0.39$; strong $0.40-0.49$; very strong 0.70 and higher.[19]

We used the Students t-test to compare the patients included in this study with the original cohort. The Chi-square test was used to compare categorical variables of interest which 
included satisfaction with treatment (excellent or good vs. fair, poor or fail) and satisfactory symptom relief (better vs. same or worse), changes in pain intensity ( $230 \%$ and $\geq 50 \%$ improvement vs. $<30 \%$ and $<50 \%$ improvement) and interference with daily activities (yes or no) and changes in FDI scores $230 \%$ improvement vs. $<30 \%$ improvement). Bivariate correlation was used to measure the association between mean pain intensity and global assessment questions. Statistical level of significance was set at 0.05. All statistical tests were performed using SPSS version 12. (SPSS Chicago, IL)

\section{Results}

Eighty-two out of 90 children completed the clinical trial and responded to the global questions that measured the primary outcome at the end of the study. As the results of the clinical trial showed no significant difference in efficacy between drug and placebo we combined the available data from children in both treatment arms for this analysis. Seventytwo out of 82 children had all the required data points of AP intensity to be included in the current study. Seven patients who had not completed the FDI questionnaire were excluded from the FDI analysis. The age and gender distribution and AP intensity of children included in the current study $(12.6 \pm 2.8$ years, $72 \%$ female, mean AP intensity $47.9 \pm 16.3 \mathrm{~mm}$ ] were similar to those in the original study [ $12.5 \pm 2.9$ years, $71 \%$ female, mean AP intensity 48.1 $\pm 16.4 \mathrm{~mm}$ ] (Student's t-test NS). We analyzed the relationship between change in pain intensity(mean AP intensity of the first week minus mean AP intensity of the last week) with the children's response to both global questions. The majority of children who had positive outcomes using both global outcome measures had ameliorated their pain throughout the clinical trial. We found a significant association between $230 \%$ decrease in pain intensity during the four weeks of the trial and in those who were considered to have a satisfactory outcome through global assessments of satisfaction with treatment and symptom relief $(\mathrm{p}<0.05)$. (Table 1) Both global questions very strongly correlated positively with each other $(\mathrm{r}=0.79 ; \mathrm{p}<0.0001)$ and had a moderate (Satisfaction with treatment) to strong correlation (symptom relief) with a $230 \%$ improvement in AP ( $r=0.37$, and $0.42 ; \mathrm{p}<0.01$ ).

We were interested in investigating whether the primary outcome of the clinical trial would differ if the global questions were substituted by the FDA newly proposed outcome ( $230 \%$ improvement in AP). We found that the percentage of patients considered as having a positive outcome varied significantly if we use the children's response to the global questions or $230 \%$ improvement in AP (Table 2). Using the data obtained in the amitriptyline clinical trial, more children would have been considered as positive outcome through the global questions (45/72 62.5\%) (positive response to one or both questions) than through the utilization of $30 \%$ improvement of AP intensity $(37 / 72,51.4 \%)(\mathrm{p}=0.04)$. Children with positive response to the global outcome measures and those who had $\geq 30 \%$ improvement were not always the same (Figure 1). Fifteen out of $45(33 \%)$ children who had a positive outcome using the global outcome questions (good/excellent satisfaction with treatment or reported feeling better to the relief question) would have failed treatment if $\geq$ $30 \%$ improvement in AP intensity would be considered as a positive outcome. Conversely, $7 / 37$ (18.9\%) children who would be considered as having a positive outcome using $30 \%$ improvement of pain would have failed the trial using any of the global outcome measures. (Figure 1) When the subset of children who had $230 \%$ reduction in pain intensity is 
analyzed by their response to each individual global question, $27 \%$ of children who had $\geq$ $30 \%$ reduction in pain intensity would have failed the clinical trial using the symptom relief global question and $24 \%$ of children would have failed using satisfaction with treatment as outcome measure.

In order to analyze the relation between the global questions and $\geq 30 \%$ improvement in AP with a clinically meaningful outcome, we assessed the behavior of both outcome measures with children's reported difficulties to complete tasks during the 4 weeks of the study (sleep, play, school attendance) and changes in FDI scores during the study period. The association with changes in FDI scores was analyzed in relation to: positive or negative outcome through response to global questions; and $230 \%$ improvement or $<30 \%$ improvement. Satisfaction with treatment was significantly associated with ( $\mathrm{p}<0.01)$ with lack of interference with common activities by child report at the exit of the study while satisfactory symptom relief approached significance $(\mathrm{p}=0.07)$. Thirty percent improvement in AP intensity was not associated with interference with common daily activities $(\mathrm{p}=0.14)$ (Table 3) Thirty percent improvement in FDI scores was associated with satisfactory symptom relief ( $\mathrm{p}=0.009)$ but not with satisfaction with treatment $(\mathrm{p}=0.1)$ or with $\geq 30 \%$ improvement in AP $(\mathrm{p}=0.27)($ Table 4$)$.

\section{Discussion}

Our study demonstrates that the commonly used outcome measures and the measures of pain improvement proposed by the FDA behave differently. There was a statistically significant association between lack of pain interference with daily activities $(\mathrm{p}<0.01)$ and overall satisfaction with treatment. Satisfactory relief was associated with $\geq 30 \%$ improvement in FDI scores, a widely used measure of disability in children with AP associated-FGIDs. There was no association between the FDA proposed clinical endpoint and pain interference with activities or $230 \%$ improvement in FDI scores. These results may not be surprising as a global assessment question should correlate with general measures of functioning. However, they do suggest that the use of pain outcomes as an exclusive measure is of limited use in children. Global and pain oriented measures seem to assess different biopsychosocial factors, an important concept to be further investigated before changes in pediatric outcomes are to be recommended. Global endpoints may not only reflect the children's perception of daily pain. Global endpoints may assess additional constructs providing a more comprehensive assessment of the child's illness experience than a measure exclusively based on pain intensity. Although intuitively logical, to the best of our knowledge this has not been previously investigated in children with AP. The fact that improvement in somatization, depression and anxiety scores paralleled the improvement of global measures in the original clinical trial may indicate that global measures also reflect changes on these psychological variables.

Changes in pain intensity are probably more difficult to conceptualize by children. Children's inability to accurately recall pain episodes within days of their occurrence [10, $20,21]$ and the lack of correlation between the intensity of those episodes with their recall suggests that accurate assessment of AP intensity may be a difficult task for children. The inability to attend school, play and sleep may be more important and meaningful to children 
than pain levels that are more variable and difficult to measure. Difficulties completing daily activities may represent a more vivid experience for the children than an abstract conceptualization of pain. It is possible that although children may not accurately recall each of the episodes of pain, they may be able to adequately conceptualize their pain experience and the negative connotations of pain reflected by their limitations in carrying out common activities. Inability to complete activities is also important to parents. School absenteeism is associated with medical consultation. Parents are usually more concerned by the child inability to complete activities due to pain than by subjective reports of pain. [3].

The results of our study suggest that the substitution of global outcomes by changes in pain intensity should not be recommended. Global outcomes have been widely used for several years and seem to reflect domains that may not be assessed by more focused pain measures. However, the results of our study do not allow us to conclude that the exclusive use of global outcome measures should be recommended either. Global assessments may be too broad and may fail to capture more subtle changes in AP. They are also subject to unconscious manipulation by patients who may choose a socially desirable answer to avoid disappointing a physician they have a close relationship with. Although we have found that most children who had a positive outcome using $\mathbf{2 3 0 \%}$ improvement in pain intensity would be accounted for using both global measures, there is a subgroup of children that would be missed if only global measures were to be used (Figure 1). The use of a combination of global and pain measures in a clinical trial would limit these shortcomings and increase the sensitivity of our outcome measures. The use of a combination of global and pain outcomes should not change the conclusion of the trial as it would affect both arms (drug and placebo) equally. It would help individualize treatment choices as children likely have differences in the measures that are relevant to them.

Our recommendation of using multiple outcomes is in line with the consensus statement by the Methods, Measurement, and Pain Assessment in Clinical Trials (IMMPACT) group that recommends not only assessing outcomes of pain intensity but also sleep, physical and emotional functioning as well as global judgment of satisfaction with treatment among others[22]. Along the same lines, another consensus statement recommended the global judgment of satisfaction with treatment and the FDI as outcome measures for clinical trials on AP in children[2].

Limitations of our study include its retrospective nature, the limited sample size and the assessment of only one of the proposed FDA co-primary endpoints. However, the other coprimary endpoint, stool character, is difficult to assess in children as there are no widely accepted validated measures for this population. An important variable that we were unable to evaluate was sleep disturbances. Previous studies have shown that sleep is frequently affected in children with FGIDs.[23] This is an important variable and we cannot exclude that sleeping disturbances may weigh heavily in the overall global experience. No studies have been published on whether sleeping difficulties improve with pain relief, and if so, what degree of pain relief is needed to solve these problems.

In conclusion our study has shown that both global measures correlated strongly with each other and moderately with the FDA recommended measure of $\geq 30 \%$ improvement in AP. 
Overall satisfaction with treatment was significantly associated with child's report of interference with activities while overall satisfactory symptom relief was associated with improvement in FDI scores. The association between the FDA recommended measure of $\geq$ $30 \%$ improvement in AP did not reach significance with interference with activities or changes in FDI scores. The results of this and previous studies leads us to hypothesize that, as expected, global measures are more comprehensive measures that may not only reflect pain improvement but interference with daily activities and could also reflect changes in anxiety, depression and somatization. The newly proposed FDA outcome measures are more restrictive and may fail to identify patients who continue to suffer from the effects of AP such as interference with activities, a relevant construct associated with child's quality of life. Future prospective studies should further analyze each of the variables involved in improvement in child's life and their relation with the specific features that characterize FGIDs. Studies evaluating a combination of outcome measures in children with AP are recommended.

\section{Acknowledgments}

Funding source: None

\section{Abbreviations}
AP
Abdominal Pain
FGID
Functional gastrointestinal disorders
IBS Irritable Bowel Syndrome
PRO Patient reported outcomes
FDI Functional Disability Inventory

\section{References}

1. Longstreth GF, et al. Functional bowel disorders. Gastroenterology. 2006; 130(5):1480-91. [PubMed: 16678561]

2. McGrath PJ, et al. Core outcome domains and measures for pediatric acute and chronic/recurrent pain clinical trials: PedIMMPACT recommendations. J Pain. 2008; 9(9):771-83. [PubMed: 18562251]

3. Boey CC, Goh KL. Recurrent abdominal pain and consulting behaviour among children in a rural community in Malaysia. Dig Liver Dis. 2001; 33(2):140-4. [PubMed: 11346142]

4. Palermo TM, Kiska R. Subjective sleep disturbances in adolescents with chronic pain: relationship to daily functioning and quality of life. J Pain. 2005; 6(3):201-7. [PubMed: 15772914]

5. Camilleri M, et al. A randomized controlled clinical trial of the serotonin type 3 receptor antagonist alosetron in women with diarrhea-predominant irritable bowel syndrome. Arch Intern Med. 2001; 161(14):1733-40. [PubMed: 11485506]

6. Novick J, et al. A randomized, double-blind, placebo-controlled trial of tegaserod in female patients suffering from irritable bowel syndrome with constipation. Aliment Pharmacol Ther. 2002; 16(11): 1877-88. [PubMed: 12390096]

7. Saps M, et al. Multicenter, randomized, placebo-controlled trial of amitriptyline in children with functional gastrointestinal disorders. Gastroenterology. 2009; 137(4):1261-9. [PubMed: 19596010]

8. FDA. Guidance for Industry Irritable Bowel Syndrome - Clinical Evaluation of Products for Treatment. FDA; Silver Spring: 2010. 
9. Lane MM, et al. Reliability and validity of a modified Bristol Stool Form Scale for children. J Pediatr. 2011; 159(3):437-441 e1. [PubMed: 21489557]

10. Chogle A, et al. Accuracy of pain recall in children. J Pediatr Gastroenterol Nutr. 2012; 55(3):28891. [PubMed: 22314392]

11. Vlieger AM, et al. Hypnotherapy for children with functional abdominal pain or irritable bowel syndrome: a randomized controlled trial. Gastroenterology. 2007; 133(5):1430-6. [PubMed: 17919634]

12. Kaminski A, et al. Antidepressants for the treatment of abdominal pain-related functional gastrointestinal disorders in children and adolescents. Cochrane Database Syst Rev. 2011; (7):CD008013. [PubMed: 21735420]

13. Farrar JT, et al. Clinical importance of changes in chronic pain intensity measured on an 11-point numerical pain rating scale. Pain. 2001; 94(2):149-58. [PubMed: 11690728]

14. Walker LS, Greene JW. The functional disability inventory: measuring a neglected dimension of child health status. J Pediatr Psychol. 1991; 16(1):39-58. [PubMed: 1826329]

15. Veldhuyzen van Zanten SJ, et al. Design of treatment trials for functional gastrointestinal disorders. Gut. 1999; 45(Suppl 2):II69-77. [PubMed: 10457048]

16. Guite JW, et al. Adolescent self-perception: associations with chronic musculoskeletal pain and functional disability. J Pain. 2007; 8(5):379-86. [PubMed: 17275417]

17. Kashikar-Zuck S, et al. Depression, coping, and functional disability in juvenile primary fibromyalgia syndrome. J Pain. 2002; 3(5):412-9. [PubMed: 14622745]

18. Lynch AM, et al. Psychosocial risks for disability in children with chronic back pain. J Pain. 2006; 7(4):244-51. [PubMed: 16618468]

19. Kachigan, SK. Statistical analysis : an interdisciplinary introduction to univariate \& multivariate methods. Vol. xvii. New York: Radius Press; 1986. p. 589

20. Lewandowski AS, et al. Comparing diary and retrospective reports of pain and activity restriction in children and adolescents with chronic pain conditions. Clin J Pain. 2009; 25(4):299-306. [PubMed: 19590478]

21. Reznik M, Sharif I, Ozuah PO. Classifying asthma severity: prospective symptom diary or retrospective symptom recall? J Adolesc Health. 2005; 36(6):537-8. [PubMed: 15901521]

22. Dworkin RH, et al. Interpreting the clinical importance of treatment outcomes in chronic pain clinical trials: IMMPACT recommendations. J Pain. 2008; 9(2):105-21. [PubMed: 18055266]

23. Iovino $\mathrm{P}$, et al. Irritable bowel syndrome in childhood: visceral hypersensitivity and psychosocial aspects. Neurogastroenterol Motil. 2009; 21(9):940-e74. [PubMed: 19368655] 


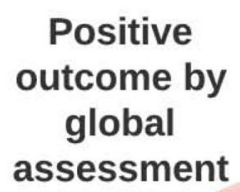

Positive outcome by change in pain intensity

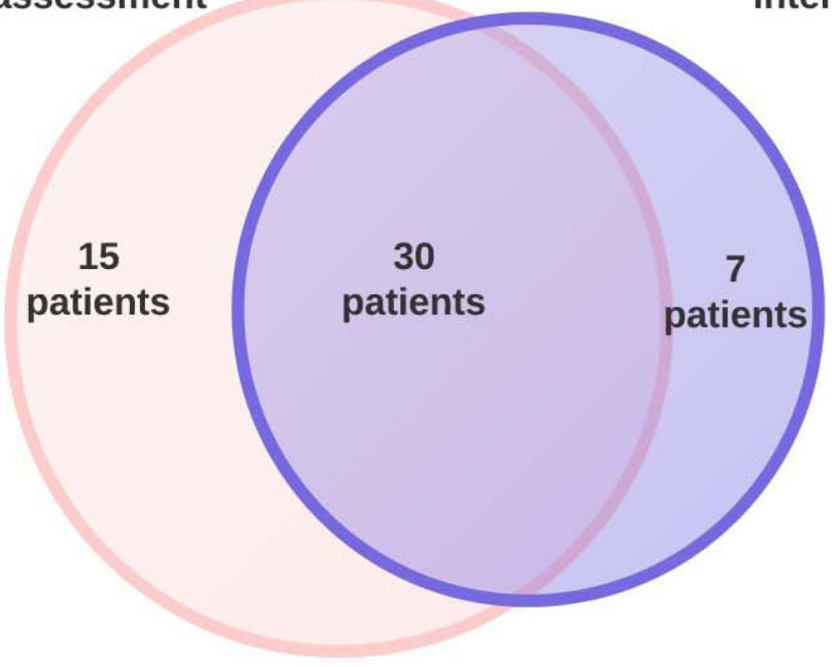

Figure 1.

Venn diagram describing relationship between patients with positive outcomes by global assessments and by $\geq 30 \%$ change in pain intensity. 


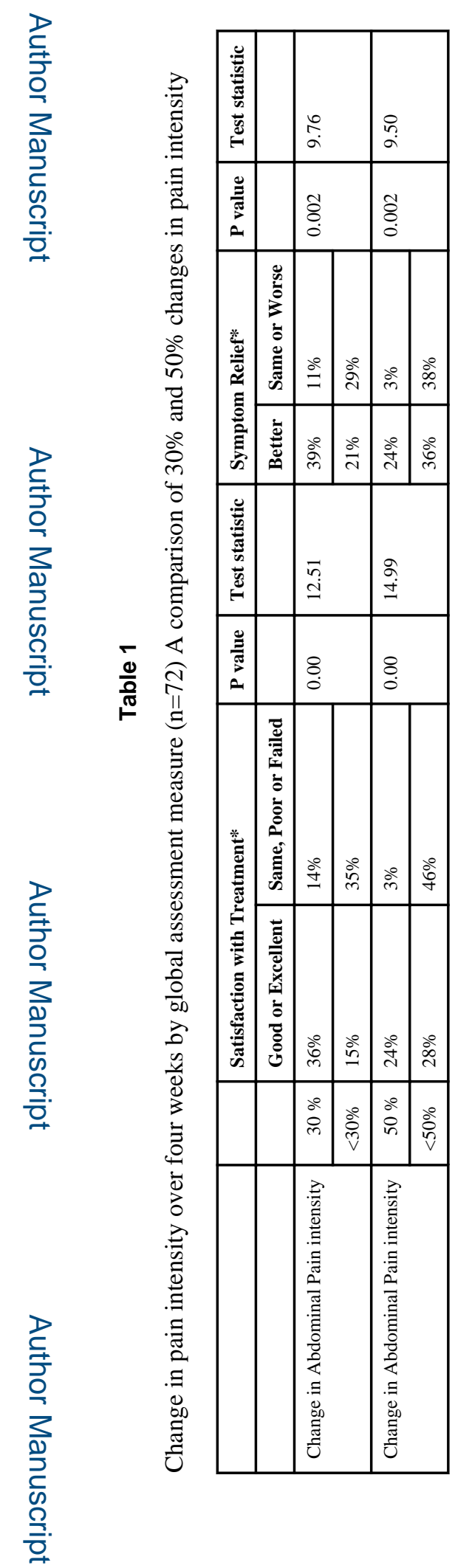

J Pediatr Gastroenterol Nutr. Author manuscript; available in PMC 2015 November 11. 


\section{Table 2}

\section{Percentage of children with positive outcomes by outcome measure}

\begin{tabular}{|c|c|}
\hline Treatment Satisfaction (Good or Excellent) & $60.2 \%{ }^{*}$ \\
\hline Symptom Relief (Better) & $48.2 \%{ }^{*}$ \\
\hline Pain Intensity ( $230 \%$ improvement) & $51.4 \%$ \\
\hline FDI ${ }^{\text {Il }}(\geq 30 \%$ improvement) & $56.9 \%$ \\
\hline \multirow{2}{*}{ p $<0.05$ compared to $230 \%$ improvement in Pain intensity } \\
II -Functional Disability Inventory
\end{tabular}

Analysis conducted with binary outcomes. Positive clinical endpoints are depicted below each outcome measure. 


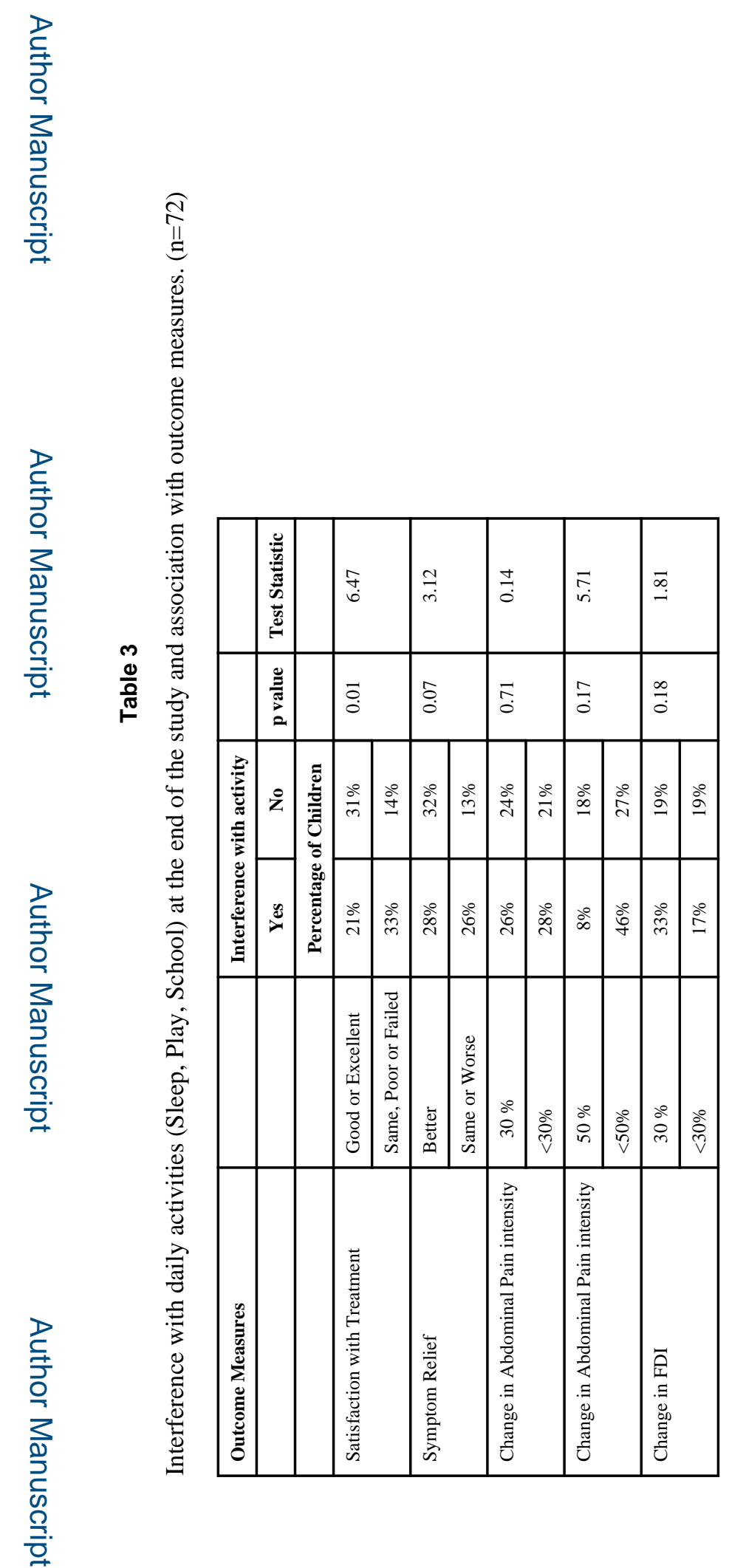

J Pediatr Gastroenterol Nutr. Author manuscript; available in PMC 2015 November 11. 


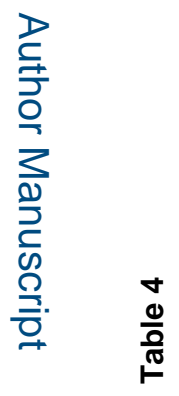

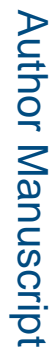

\begin{tabular}{|c|c|c|c|c|c|c|c|c|c|c|}
\hline 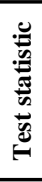 & & & \multicolumn{2}{|l|}{$\tilde{\vec{i}}$} & \multicolumn{2}{|l|}{$\underbrace{\hat{\sigma}}_{6}$} & \multicolumn{2}{|l|}{$\stackrel{\vec{J}}{\rightarrow}$} & \multicolumn{2}{|l|}{ 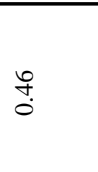 } \\
\hline 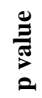 & & & $\stackrel{m}{0}$ & & ठ̊. & & $\hat{\widehat{c}}$ & & in & \\
\hline \multirow{3}{*}{ 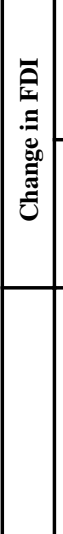 } & $\stackrel{0}{0}$ & 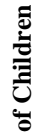 & $\frac{\circ}{m}$ & $\stackrel{\sim}{\sim}$ & $\begin{array}{l}80 \\
8 \\
b\end{array}$ & $\stackrel{\circ}{\circ}$ & $\frac{\stackrel{\circ}{2}}{v^{2}}$ & ¿̊ㅇ & $\stackrel{\infty}{\infty}$ & ণั \\
\hline & $\stackrel{\circ}{0}$ & 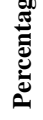 & $\stackrel{8}{\stackrel{2}{1}}$ & $\stackrel{\sim}{\sim}$ & $\stackrel{\circ}{\Xi}$ & $\stackrel{\sim}{\ddot{\sim}}$ & $\stackrel{\circ}{\Xi}$ & $\stackrel{\circ}{\check{~}}$ & $\check{a}^{\circ}$ & $\frac{\stackrel{\circ}{m}}{m}$ \\
\hline & & & 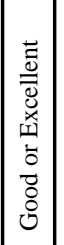 & 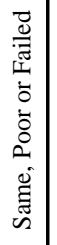 & 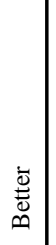 & 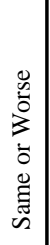 & $\begin{array}{l}\therefore \\
\stackrel{x}{1} \\
\therefore\end{array}$ & $\begin{array}{l}\stackrel{0}{0} \\
\bar{v}\end{array}$ & $\begin{array}{l}0 \\
0 \\
\text { h }\end{array}$ & 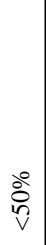 \\
\hline 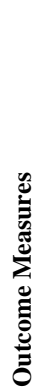 & & & 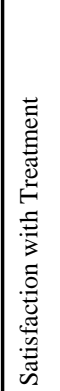 & & 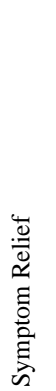 & & 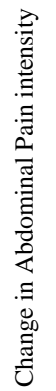 & & 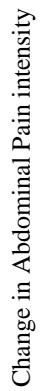 & \\
\hline
\end{tabular}

J Pediatr Gastroenterol Nutr. Author manuscript; available in PMC 2015 November 11. 